\title{
Route Hitting Probability for a Class of Ad Hoc Routing Protocols
}

\author{
Cedric Westphal \\ DoCoMo Labs USA \\ cwestphal@docomolabs-usa.com
}

\author{
Charles Perkins \\ Nokia-Siemens-Networks \\ charles.perkins@nsn.com
}

\author{
Ryuji Wakikawa \\ Keio University \\ ryuji@sfc.wide.ad.jp
}

\begin{abstract}
We compute the probability that a path is discovered by a class of reactive routing protocols which we denote as random reactive protocols. These reactive protocols do not flood the network, but attempt to find a path from the source to the destination by sending a packet to a destination chosen randomly. Several protocols, including VRR or AODV-NF can be included in this class.

We compute the route hitting probability for such packet, namely the probability that the packet will encounter a node which has a path to the destination. We analytically model the performance of a route discovery scheme which does not rely on flooding to find the connection destination, and show that such system is theoretically promising.
\end{abstract}

Index Terms-Wireless ad hoc networks, route discovery, Virtual Ring Routing.

\section{INTRODUCTION}

Reactive routing protocols in mobile ad hoc networks (MANETs) function in two steps: the first one is a control phase to locate the corresponding node, and the second involves the actual transmission of the data. Many protocols (say, [1], [2], [3], [4], [5]) suggest to use flooding to locate the destination. However, flooding is detrimental for many reasons: it requires the global involvement of all nodes in the network, and even locally, the competition and congestion at the MAC layer creates the so-called broadcast storm [6].

Because flooding has such adverse effects, many reactive protocols have tried to minimize the impact of flooding. Some protocols suggest to cache the discovered routes, so as to reuse the cached routes instead of flooding the network for the subsequent connections. Some protocols propose alternative to the flooding mechanism to locate the destination.

One such alternative is to unicast a route discovery packet to a point with certain properties, in the hope of encountering along the way a node with a path to the destination. The properties of the endpoint for the initial route discovery vary with the protocol. We will describe several protocols which belong to this general category. However, in general, this endpoint can be described as random with respect to the source and the destination.

The route discovery thus turns into a geometric probability problem: if the mobility ensures that the nodes are uniformly distributed over the area of the ad hoc network, finding a route to the destination translates into a random path issued from the source intersecting with a random path which knows where the destination is. We characterize the probability that a random

This research was sponsored in part by a grant from the Japan Society for Promotion of Science (JSPS). route request finds a path which knows where the destination is, which we call the route hitting probability.

We show that the route hitting probability is relatively low in general, at about $23 \%$, but iterating the route discovery process increase the probability of route discovery significantly.

This document is organized as follows. We first describe two reactive routing protocols which belong to this category, Virtual Ring Routing, VRR [7] and AODV-NF [8], and some related work in Section II. Then we compute the route hitting probability in a circle and in $\mathrm{n}$-sided regular polygons in Section III. We conclude the work with some future research directions in Section IV.

\section{RELATED WORK}

Much work has gone into analyzing reactive routing protocols and in suggesting improvements for the two reactive protocols, AODV [1] and DSR [2], which have been standardized as experimental RFCs in the IETF. AODV-PA [9] suggested the use of path accumulation in order to increase the effectiveness of flooding as a means of distributing routing information. This has been proven [10] to reduce the need for flooding in subsequent requests.

Another idea to mitigate the broadcast storm [6] which accompanies flooding is to regulate the broadcast. Constructing a Connected Dominating Set (CDS) to use as a broadcast backbone has been studied in for instance [11], [12] and some proposals have made it into IETF drafts [12]. However, while the benefits of a broadcast backbone are obvious in order to reduce the competition between subsequent retransmissions, it creates the need to manage such a backbone in a dynamic environment. It also creates inequality in the role of the different nodes, those which belong to the CDS and those who are leaf nodes. The path discovery must discover routes constrained to the CDS. This leads to imbalance in the traffic distribution over the network, to potential bottlenecks over the CDS and to unfairness in the power consumption.

Gossip protocols [13], [14] avoid the route discovery phase by sending a route discovery message which is probabilistically forwarded at each node. However, such protocols might not find the destination and the gossip packet might run into routing loops. Furthermore, there is no bound on the delay to find a route.

Another tack to alleviate the issue of broadcast has been to use geographical routing and last encounter routing [15]. [16] uses spatio-temporal correlation to minimize the route discovery process by searching for nodes which have met the destination more recently than the source of the route request. 
These protocols loosely fit in our reactive ad hoc routing category, as we will describe in Section IV.

[17] describes a routing protocol which avoids the local minimum issue common to geographic forwarding, by inserting randomness in a geographic routing protocol. It is tangentially related to the protocols we study below, which use randomness as well to locate the destination.

We consider a category of reactive protocols, which issue RREQ to a point which is randomly distributed in the network area, hoping to find a path to the destination by traversing the network between the source and this random point. We call these protocols random reactive protocols.

\section{A. Virtual Ring Routing}

Virtual Ring Routing (VRR) [7] does away with flooding altogether, and this protocol fits in our class of random reactive protocols. We will describe the protocol in more details.

VRR imposes a routing overlay on the network, akin to a distributed hash table. Nodes need to maintain a path to $r$ predetermined neighbors at all times, in order to distribute some routing information through the network.

This is constructed as follows: all nodes have a random integer assigned to them, which is used as an identifier. Without loss of generality, we can assume we have $n$ nodes, and that each node is randomly a identifier in $\{0,1, \ldots, n-1\}$ Each nodes $k$ maintains a path to nodes $k+1, \ldots, k+r / 2$ and $k-1, \ldots, k-r / 2$ (we assume $r$ is even, and all operations are modulo $n$ ). These $r$ nodes are the "virtual neighbors" of $k$ and define the virtual set (vset) of $k$. Because all the operations are modulo $n$, this induces a ring topology.

When node $s$ wants to send a packet to node $d$, it does so by sending the packet to the node which minimizes the distance $(d-k) \equiv n$ for all $k$ in the route table of $s$. This means that the packet will travel around the virtual ring. The ring insures correctness of the routing: a message will always eventually find its destination (ignoring losses at the MAC layer or transient inconsistencies), in the worst case going all the way around the ring.

One hop on the virtual ring corresponds to traversing a physical path in the actual network between the two virtual neighbors. The protocol includes the following provision: if the physical path in the actual network encounters a node which has $d$ in its route table, then the message has found a path to the destination. It stops its forwarding along the virtual ring, and goes instead to $d$.

\section{B. $A O D V-N F$}

Maintaining a virtual ring overlay could be cumbersome in a network where nodes come and go. The AODV-NF protocol was proposed which does not require to maintain any routing overlay, based on AODV. AODV distributes route information throughout the network every time it floods a route request. All nodes which receive the route request (RREQ) insert a route to the source of the route request in their route table, and keep this route for a length of time denoted the Active Route Time-out. Further, AODV can be enhanced to support path accumulation (AODV-PA [9]), in which each node which receives the route request inserts not only the source of the RREQ, but also all the intermediary points between the source and the node in its route table.

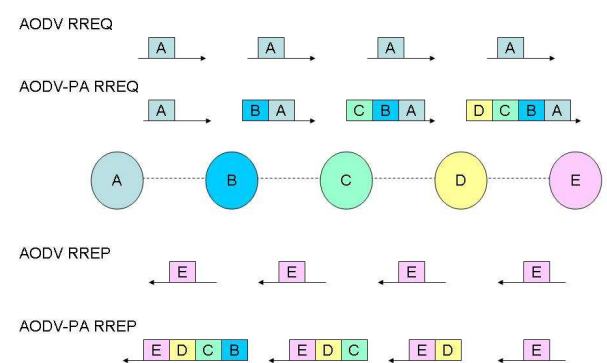

Fig. 1. Path Accumulation Mechanism for Route Discovery

Depending on the rate at which RREQ are issued and the length they are kept, this mechanism disseminates some amount of route information into the network. AODV-NF attempts to make use of this information in the following manner: when node $s$ has a packet connection which requires to send a RREQ, $s$ looks up its route table. If the number of distinct routes in the table is above a threshold, it picks one node $k$ at random and unicasts the RREQ to this node. The packet is then routed towards $k$. If along the way, it encounters a node which has an active path to $d$, then this node sends the route reply (RREP) to $s$. If the node reaches $k$ without encountering such path, $k$ either repeats the procedure, or sends a negative acknowledgment to $s$, which then repeats the procedure with a different point. The process is repeated for some number of time. If no path is found after a given number of iterations, then AODV-NF then defaults to AODV and floods the network.

\section{Last Encounter Routing}

While Last Encounter Routing [15] does not exactly fit in our routing protocol category, it may offer some insight to describe it. Each node in [15] keeps tab of the last time and location it has met with every other nodes in a mobile network. When $s$ needs to send a packet to $d$, it looks up the last location when $d$ and $s$ were in contact together, and the time $\tau$ since $d$ and $s$ have met. It then sends the packet to the node $k$ nearest that location. If along the way, it does not encounter a node which has met $d$ within time $\tau / 2, k$ initiates an expanding ring search to locate such a node. When such a node is found, the procedure is repeated, with a new value $\tau^{\prime}$.

While VRR and AODV-NF first pick a node randomly, LER does not. However, if the time scale for the mobility is such that the position of $k$ and $s$ are no longer correlated, then $s$ and $k$ become two points randomly distributed in the network area. The concept of a path to the destination is also different in the LER setting. One can consider some coherence time $\theta$, and two regimes roughly defined as: if node $j$ has met $d$ 
within time $\theta$, then it can reconstruct the path by following the time gradient induced by LER; if node $j$ has not met $d$ within time $\theta$, then the positions of $d$ and $j$ are now independent. The protocol thus can be approximated as sending a packet on a random path, hoping to find a path to the destination; if not, an expanding ring search is performed to find a new starting point, and the process iterates.

\section{Route Hitting Probability}

We attempt here to answer the following question: what is the chance that a random reactive protocol finds a path to the destination in its first try? We consider in this section a geometric abstraction of the problem and conduct some analysis in order to find the route hitting probability.

\section{A. Unit disk area}

We consider a disk area populated with sufficiently many nodes so that we can approximate the shortest path as a straight line.

Assume further that some knowledge of the network topology has been distributed throughout the network, in such a way that, for a path between two end points, all the intermediate nodes on the path are able to locate these two end points. This is a reasonable assumption, as DSR or AODV-PA satisfy this requirement. Assume that for any possible destination, there are at least $P$ paths which know where the destination is. Further, assume that the RREQ process is uniformly distributed over the area, meaning that, for any connection, a source $S$ and a destination $D$ are chosen randomly according to a uniform distribution over the area. Similarly, the $P$ end points of the path to the destination, denoted $P_{i}$ for $i=1$ to $P$, follow the same uniform distribution. We call the $P$ paths defined thusly as destination-aware paths.

We are interested in assessing the performance of the following scheme: since there exist at least $P$ paths to any destination, then conversely, any nodes will know the path to at least $P$ other points. For any source $S$, pick a point $I_{1}$. The RREQ will follow the path $\left(S I_{1}\right)$. If it crosses any one of the paths $\left(D P_{i}\right), i=1, . ., P$, then denote by $X$ the intersection of the two segments. $X$ is the cross-over point. The path $S X D$ is a path from the source to the destination.

It is of course not optimal. For instance, figure 2 describes one such process (generated using MatLab) where the path stretch (that is, the ratio of the path $S X D$ over the length $S D$ is 1.55 . However, the key trade-off here is in the reduction of the route discovery overhead: in the case of Figure 2, the overhead is very little: the only nodes involved in the route discovery process are the nodes on the path $S X D$.

We now attempt to formalize this trade-off mathematically. We first assume that $P=1$ (we will discuss other values of $P$ subsequently).

Define the points $A_{1}, A_{2}, A_{3}$ as the respective intersections of the boundary circle with the half-lines $[S D),\left[D P_{1}\right)$ and $\left[P_{1} S\right)$, and by $B_{1}, B_{2}, B_{3}$ the respective intersection of the boundary with the half-lines $\left[P_{1} D\right),\left[S P_{1}\right)$ and $[D S)$. Denote by $\mathcal{B}_{1}, \mathcal{B}_{2}$ and $\mathcal{B}_{3}$ the area respectively defined by the sectors

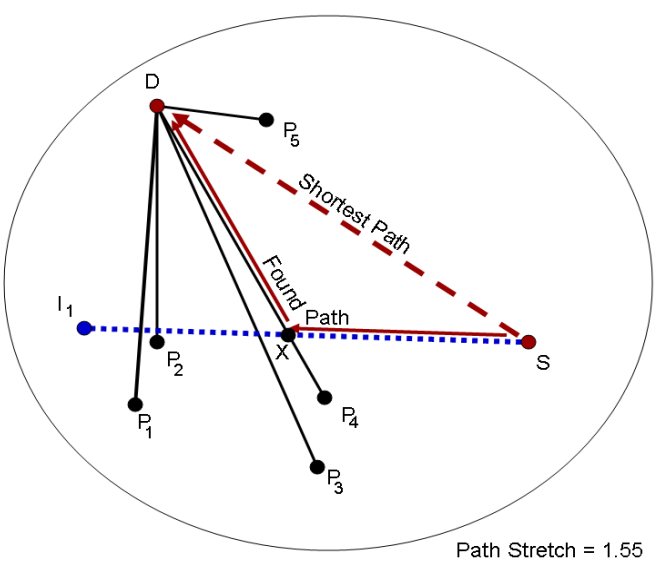

Fig. 2. Model Description: A Source Finds a Path with Stretch 1.55 to the Destination

$\widehat{A_{1} \widehat{D B}} B_{1}, A_{2} \widehat{P_{1}} B_{2}$ and $\widehat{A_{3} \widehat{S B}}{ }_{3}$ as they intersect the network area. See Figure 3 for a pictorial description of the set-up. Denote by $\mathcal{A}_{1}$ the area defined by the sector $\widehat{A_{1}} \widehat{D A_{2}}$ minus $\mathcal{B}_{1}$. Similarly, $\mathcal{A}_{2}$ is $\widehat{A_{2}} \widehat{P_{1}} A_{3} \backslash \mathcal{B}_{2}$ and $\mathcal{A}_{3}$ is $\widehat{A_{3} S A_{1}} \backslash \mathcal{B}_{3}$.

Theorem 3.1: The probability $p_{1}$ that a unicast route request $S I_{1}$ intersects a single destination-aware path $D P_{1}$ is equal to:

$$
p_{1}=\frac{1}{3}\left(1-\frac{35}{12 \pi^{2}}\right)=23 \%
$$

Proof: The probability that the path $S I_{1}$ and $D P_{1}$ intersect is equal to the probability that $I_{1}$ is in the area $\mathcal{A}_{1}$. This is equal to one third of the probability that $I_{1}$ belongs to any of the $\mathcal{A}_{i}$ areas, since the $\mathcal{A}_{i}$ follow the same distribution by construction. The probability that a uniformly distributed point falls into any $\mathcal{A}_{i}$ is equal to the probability that the points form a convex quadrilateral. Solomon [18] shows that the probability that the four points do not form a convex quadrilateral, that is the probability that $I_{1}$ is in any $\mathcal{B}_{i}$ or in the triangle $S D P_{1}$, is equal to $\frac{35}{12 \pi^{2}}$. Note that a similar proof was given in a totally different security context by Parno et al. [19].

\section{B. Extended Destination-Aware Path}

We now assume that the path actually extends beyond $P_{i}$ and reaches the boundary of the area. Thus the path which knows the location of the destination is the half-line $\left[D P_{1}\right)$, namely the segment $\left[D A_{2}\right]$. We consider this case, as paths to $D$ are primarily distributed by the RREQs from $D$, and most of these only stop at the boundary ${ }^{1}$.

The unicast request traveling on the path $\left(S I_{1}\right)$ will encounter the line $D A_{2}$ if and only if $I_{1} \in \mathcal{A}_{1} \cup \mathcal{B}_{1}$. Since $I_{1}$ is distributed uniformly, it will belong there with probability equal to the size of the area (recall the overall area is a unit disk). Since we have chosen $S, D$ and $P_{1}$ distributed

\footnotetext{
${ }^{1}$ Even as we are trying to minimize the number of RREQs, we still want some to be broadcast, if only for the case where unicast requests do not find a destination aware path.
} 
uniformly, the surfaces of the area $\mathcal{A}_{i} \cup \mathcal{B}_{i}, i=1,2,3$ follow the same distribution, and thus the average of the area of $\mathcal{A}_{1} \cup \mathcal{B}_{1}$ is equal to that of $\mathcal{A}_{2} \cup \mathcal{B}_{2}$ and $\mathcal{A}_{3} \cup \mathcal{B}_{3}$.

Thus, in average, the size of the area $\mathcal{A}_{1} \cup \mathcal{B}_{1}$ is equal to one third of the area outside of the triangle $D S P_{1}$, which is equal to one third of one minus the area of the triangle. It is a classical result in geometric probability to compute the area of such a triangle in the unit circle (see for instance Blaschke [20]), and it is equal to $\frac{35}{48 \pi^{2}}$. Thus we can state the following theorem:

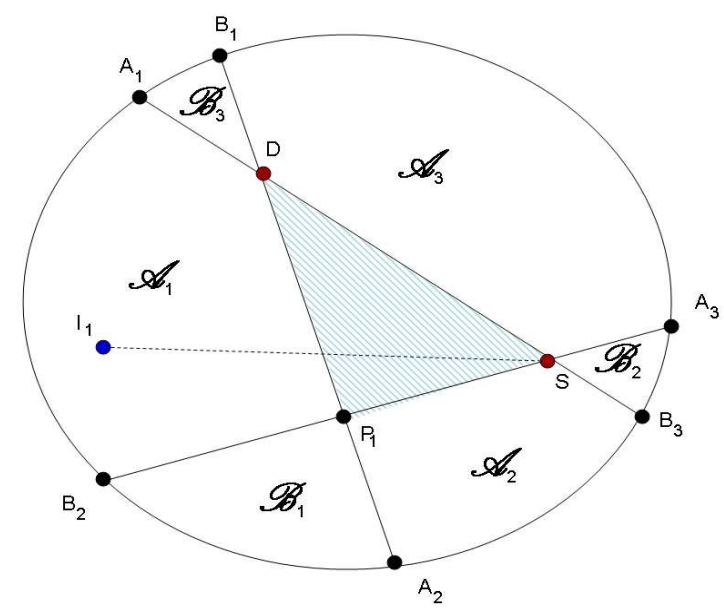

Fig. 3. Different areas $\mathcal{A}_{i}, \mathcal{B}_{i}, i=1,2,3$ defined by the points $S, D, P_{1}$ and $I_{1}$

Theorem 3.2: The probability $p_{2}$ that a unicast RREQ $S I_{1}$ encounters a single destination-aware path issued from the destination to the boundary of the area $D A_{2}$ is equal to:

$$
p_{2}=\frac{1}{3}\left(1-\frac{35}{48 \pi^{2}}\right)=0.3087
$$

Remark 3.1: This probability is the same if the destinationaware path is the line segment $\left[D P_{1}\right]$ and the source picks first a random destination $I_{1}$, then looks up for the furthest node in its route table for which a path goes through $I_{1}$. The RREQ then travels on the half-line $\left[S I_{1}\right)$ and attempts to hit the line segment $\left[D P_{1}\right]$. It makes sense to look for the furthest node, as it increases the chance of crossing a destination-aware path. The proof follows from the same geometric observations.

\section{Areas of Different Shape}

We can extend the result to other regular network areas, instead of a unit circle. We still consider the extended destination aware path.

Theorem 3.3: Define $\omega=\frac{2 \pi}{n}$. For a unit-size $n$-gon, the probability $p_{n}$ that a unicast RREQ $S I_{1}$ encounters a single destination-aware path issued from the destination to the boundary of the area $D A_{2}$ is equal to:

$$
p_{n}=\frac{1}{3}\left(1-\frac{9 \cos ^{2} \omega+52 \cos \omega+44}{36 n^{2} \sin ^{2} \omega}\right)
$$

Proof: The proof is similar to the proof of the circular area, with the difference in computing the area of the triangle $S D P_{1}$ inside a unit $n$-gon instead of a unit disk. The average area $\mathcal{T}_{n}$ of the triangle picked randomly inside the $n$-gon is given by Alikoski's formula [21]:

$$
\mathcal{T}_{n}=\frac{9 \cos ^{2} \omega+52 \cos \omega+44}{36 n^{2} \sin ^{2} \omega}
$$

For instance, for a unit square area, the probability of a hit is equal to $\frac{1}{3}\left(1-\frac{11}{144}\right)=0.3079$. The difference between the hit probability for regular $n$-gons is relatively small, since it will increase from the value for the unit square area and converge as $n \rightarrow \infty$ to the value for the unit circle area, and both values can be rounded to $31 \%$ when considering only 2 digits accuracy.

We expect a similar range for other convex areas.

\section{Multiple Paths and Relays}

We have so far considered the case of a single route request and a single destination-aware path. Since multiple destination-aware paths intersect at the destination, they are not independent. Thus it is difficult to compute a closed form expression for the probability that the route request finds a path if there are several destination-aware path, or if it is forwarded several times from $S$ to $I_{1}$ to $I_{2}$ to $I_{3}$, and so on.

In this section, we present some Matlab results which depict the performance of our geometric abstraction in order to give us sound guidelines for the design of the protocol.

We compute the probability that a unicast route-request hits one of $P$ destination-aware path. We plot the probability of hitting one of the destination-aware path against the number of path $P$ on Figure 4 for both a circular unit area and a square one. Note that the first value $P=1$ confirms the value that we analytically derived. We also plot the probability of hitting a destination-aware path that extends all the way to the boundary (that is $D A_{2}$ according to our terminology) both in a unit disk and a unit square. Finally, we plot on the same graph the probability of hitting a destination-aware path $D A_{2}$ when the RREQ from $S$ to $I_{1}$ is assumed to be forwarded all the way to the boundary.

We now consider the following mechanism: if $I_{1}$ receives a unicast RREQ from $S$ which has not encountered a path to the destination $D$ along the way, then, if $I_{1}$ does not know where $D$ is, it resends the RREQ as a unicast message to another random point $I_{2}$. The sequence is build iteratively, up to the $I$ th relay, where $I$ is the maximum number of unicast RREQs that we allow. For now, we set this maximum value to $\infty$ and we compute the cumulative distribution function of the probability that the the RREQ disseminated this way hits a destination-aware path. The probability that one finds a destination aware path in less than $k$ relays is plotted on Figure 5.

We also consider the case of the sender $D$ sending multiple unicast RREQs to several intermediate points $I_{j}$. That is, in the case that no destination aware path is encountered, instead of having $I_{1}$ send the RREQ to $I_{2}$, it is $D$ who sends it to $I_{2}$, 


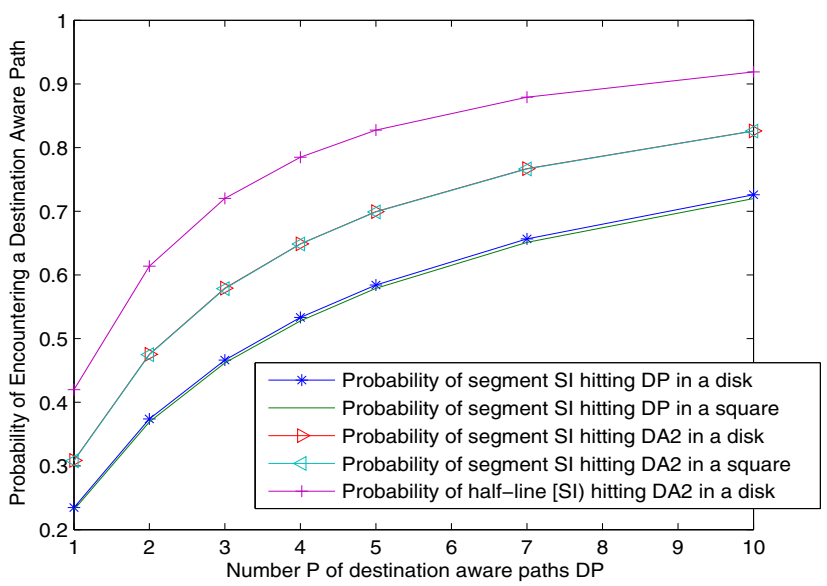

Fig. 4. Probability that a Unicast RREQ Hits one of $P$ Paths.

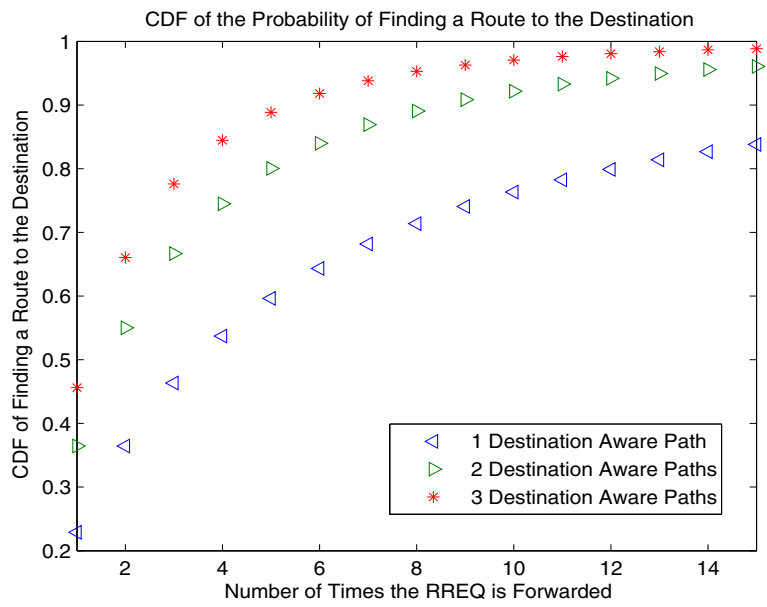

Fig. 5. Cumulative Probability of a Hit vs. the Number $I$ of Relaying Intermediate Points

where $I_{2}$ is chosen uniformly distributed over the unit area. This is depicted by Figure 6 . One can see that the probability of finding a destination aware path is roughly similar by using a RREQ which goes from $S$ to $I_{1}$ to $I_{2}$, etc, or by using a RREQ going $S$ to $I_{1}, S$ to $I_{2}$, and so on.

Lastly, we consider the length of the path found this way. Figure 7 shows the path stretch, that is the total path length divided by the shortest path, for different number $i$ of unicast RREQs $S \rightarrow I_{i}$ and different number of destination aware paths. While suboptimal, the path stretch remains quite reasonable under our modeling assumptions.

\section{CONCLUSION}

The conclusion of this geometric analysis is that for a protocol to be successful, the number of destination-aware paths should be kept to a certain threshold, and the number of intermediate points $I_{i}$ can be upper bounded by a small value as well.

For instance, the number $r$ of virtual elements in virtual set

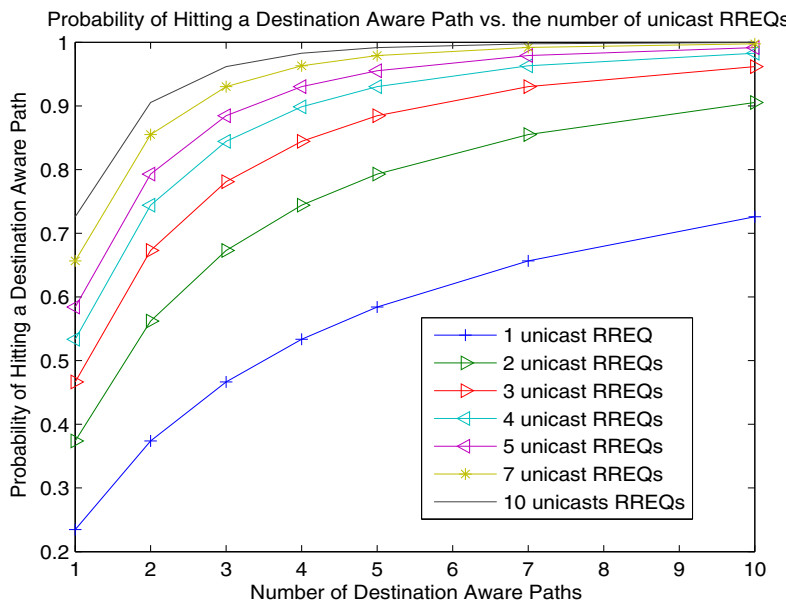

Fig. 6. Probability of a Hit vs. the Number of Paths from the Source to an Intermediate Points

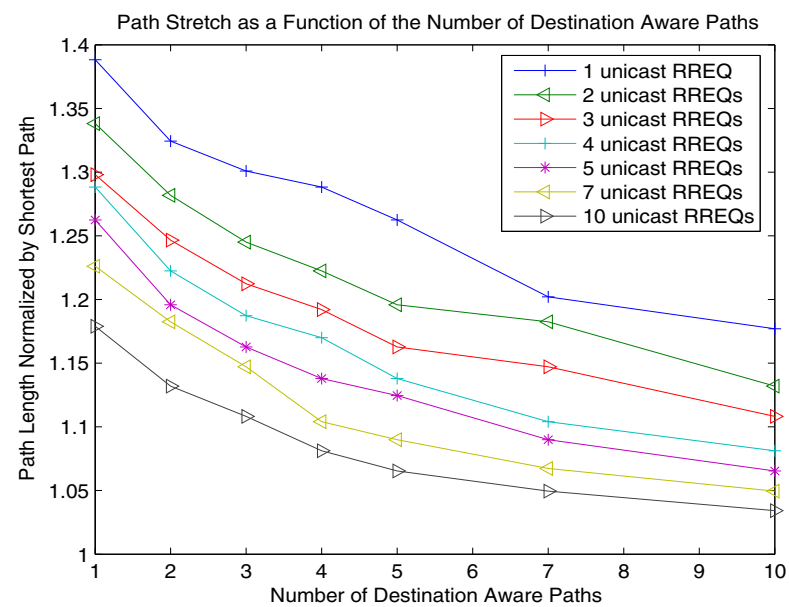

Fig. 7. Path Length of the Path Found Using the Unicast RREQ

vset in VRR is directly related to the route hitting probability. Similarly, the route hitting probability will be related to the threshold at which to send a unicast RREQ in AODV-NF. The question becomes: what is the minimal set of virtual neighbors (for VRR), or what is the lowest threshold (for AODV-NF), for which the route hitting probability is high enough to deliver reasonable performance. The answer to this question reduces the overhead, since it requires to maintain the smallest virtual neighbor set in VRR, or to send the least amount of flooded RREQ in AODV-NF. The optimization of $r$ (or of the AODV$\mathrm{NF}$ threshold) with respect to some performance criteria is an interesting area for future research.

The results here are straightforward, as the first step of the route discovery process can be modeled by points which have position independent of each other. However, the first path $\left(S I_{1}\right)$ traversing the network which does not encounter a destination aware path $(P D)$ conditions negatively the probability that $D$ is in the vicinity of $I_{1}$.

This means that, for VRR, the analysis even in our simpli- 
fied framework, becomes difficult. Assuming that $I_{1}$ is now the source looking for $D$ and computing the route hitting probability will give a probability which is intuitively less than for $S I_{1}$. By symmetry, this has the same effect on $S$ : assuming that $S$ sends the request to $I_{2}$ should yield the same probability.

If we compare our results to the simulations in [7], we see that VRR is more efficient to discover the route than our model predicts. However, this is due to the simulation set-up, which consists of a network of size $300 \mathrm{~m} \times 1,500 \mathrm{~m}$ with a transmission range of $250 \mathrm{~m}$. This means that the area is not a regular polygon, as in our model, but degenerates into a linear network.

A study of the different iterations of the route hitting probability offers an interesting challenge for future research. More generally, the geometric distribution of routing information in an ad hoc network reveals a lot of interesting problems.

\section{REFERENCES}

[1] Charles E. Perkins, Elizabeth M. Belding-Royer, and Samir Das. Ad Hoc On Demand Distance Vector (AODV) Routing. IETF RFC 3561.

[2] David Johnson, David Maltz and Yih-Chun Hu, The Dynamic Source Routing Protocol for Mobile Ad Hoc Networks (DSR), IETF MANET working group, draft-ietf-manet-dsr-10.txt, work in progress, July 2004.

[3] Charles Perkins, Elizabeth M. Royer. Ad hoc On-Demand Distance Vector Routing. Proceedings of the 2nd IEEE Workshop on Mobile Computing Systems and Applications, New Orleans, LA, February 1999, pp. 90-100.

[4] Ian Chakeres, Charles Perkins, Dynamic MANET On-demand Routing Protocol. IETF Internet Draft, draft-ietf-manet-dymo-06.txt, October 2006 (Work in Progress).

[5] H. Rangarajan, J.J. Garcia-Luna-Aceves, Using Labeled Paths for Loopfree On-Demand Routing in Ad Hoc Networks, in Proc. MobiHoc'04, Tokyo, Japan, May 2004.

[6] Sze-Yao Ni, Yu-Chee Tseng, Yuh-Shyan Chen and Jang-Ping Sheu, The broadcast storm problem in a mobile ad hoc network in Proc. of the 5th annual ACM/IEEE international conference on Mobile computing and networking, pp.151-62, 1999.
[7] Matthew Caesar, Miguel Castro, Edmund Nightingale, Greg O'Shea, Antony Rowstron, Virtual Ring Routing: Network Routing Inspired by DHTs in Proc. of ACM SIGCOMM'06, Pisa, Italy, pp.351-62.

[8] Cedric Westphal, Yali Wang, Charles Perkins, Ryuji Wakikawa, Reactive Routing in Ad Hoc Networks With No Flooding, Nokia-Siemens-Network Technical Report, July 2007. http://people.nokia.net/cedric/Papers/aodvnf.pdf

[9] Sumit Gwalani, Elizabeth M. Belding-Royer, and Charles Perkins. AODVPA: AODV with Path Accumulation in Proc. of IEEE ICC'03.

[10] Karim Seada, Cedric Westphal, Charles Perkins, Analyzing Path Accumulation for Route Discovery in Ad hoc Networks, in Proc. of WCNC'07, Hong-Kong, March 2007.

[11] Bevan Das, Vaduvur Bharghavan, Routing in Ad-Hoc Networks Using Minimum Connected Dominating Sets, in Proc. of IEEE ICC'97, pp. 376-380.

[12] Joseph Macker (Editor), Simplified Multicast Forwarding for MANET, IETF draft, draft-ietf-manet-smf-05, June 2007, work in progress.

[13] Zygmunt Haas, Joseph Halpern, Li Li, Gossip-Based Ad Hoc Routing in Proc. of IEEE Infocom 2002.

[14] Stephen Boyd, Arpita Ghosh, Balaji Prabhakar, Devavrat Shah, Randomized gossip algorithms, in IEEE Transactions on Information Theory 52(6): 2508-30 (2006).

[15] Matthias Grossglauser, Martin Vetterli, Locating nodes with EASE: Last Encounter Routing for Ad Hoc Networks through Mobility Diffusion, in Proc. of Infocom 2003, San Francisco, USA.

[16] Henri Dubois-Ferrière, Matthias Grossblauser, Martin Vetterli, Age Matters: Efficient Route Discovery in Mobile Ad Hoc Networks Using Encounter Ages in Proc. of ACM MobiHoc 2003, Annapolis, USA.

[17] Sundar Subramanian, Sanjay Shakkottai, Piyush Gupta, On Optimal Geographic Routing in Wireless Networks with Holes and Non-Uniform Traffic, in Proc. of IEEE Infocom 2008, Anchorage, USA.

[18] Herbert Solomon, Geometric Probability Society for Industrial and Applied Mathematics (SIAM), 1978.

[19] Bryan Parno, Adrian Perrig and Virgil Gligor, Distributed Detection of Node Replication Attacks in Sensor Networks, in Proc. of the IEEE Symposium on Security and Privacy, Oakland, CA, May, 2005.

[20] Wilhelm Blaschke. Vorlesungen über Differentialgeometrie Vol. 2, Springer, Berlin 1923.

[21] H. A. Alikoski, Über das Sylvestersche Vierpunktproblem, in Annales Academiae Scientiarum Fennicae 51 (1938), no.7, pp.1-10. 